\title{
First-passage and first-exit times of a Bessel-like stochastic process
}

\author{
Edgar Martin,, , U Ulrich Behn,, , 田 and Guido Germano ${ }^{1,3, \text {, }}$ \\ ${ }^{1}$ Fachbereich Chemie und WZMW, Philipps-Universität Marburg, 35032 Marburg, Germany \\ ${ }^{2}$ Institut für Theoretische Physik, Universität Leipzig, 04103 Leipzig, Germany \\ ${ }^{3}$ Dipartimento SEMeQ, Università del Piemonte Orientale Amedeo Avogadro, 28100 Novara, Italy
}

\begin{abstract}
We study a stochastic process $X_{t}$ related to the Bessel and the Rayleigh processes, with various applications in physics, chemistry, biology, economics, finance and other fields. The stochastic differential equation is $d X_{t}=\left(n D / X_{t}\right) d t+\sqrt{2 D} d W_{t}$, where $W_{t}$ is the Wiener process. Due to the singularity of the drift term for $X_{t}=0$, different natures of boundary at the origin arise depending on the real parameter $n$ : entrance, exit, and regular. For each of them we calculate analytically and numerically the probability density functions of first-passage times or first-exit times. Nontrivial behaviour is observed in the case of a regular boundary.
\end{abstract}

PACS numbers: $02.50 . \mathrm{Ey}, 05.10 . \mathrm{Gg}$, 05.10.Ln, 05.40.Jc

\section{INTRODUCTION}

In the theory of stochastic processes, the first-hitting time is defined as the time when a certain condition is fulfilled by the random variable of interest for the first time; it is random itself and a particular case of a stopping time. We speak of a first-passage time when the random variable reaches a certain level for the first time, and of a first-exit time when it leaves a certain interval for the first time. A standard example of a first-passage time problem is the decision of an investor to buy or sell a stock when its fluctuating price reaches a certain threshold. However, first-passage times play an important role also in chemical physics; early examples are given by models describing the dissociation of diatomic molecules as a first-passage time problem, where dissociation occurs if a certain critical energy level is reached through collisions [1 3]. A view on diffusion in fluids based on firstpassage times has been proposed by Munakata [4], where self-diffusion is measured via the first-passage time with respect to a boundary marked by a sphere centered at the original position of a labeled particle. Problems like neuron dynamics, self-organized criticality or dynamics of spin systems can be viewed as first-passage processes in one dimension [5]. The first-passage problem is closely connected to persistence, which is the probability that a random variable does not leave a certain region up to a certain time, i.e. the complementary event to a firstpassage at the same time. The problem of persistence in spatially extended nonequilibrium systems has attracted great interest both theoretically and experimentally, see Majumdar [6] and references included therein, where persistence is defined as the probability that for an arbitrary nonequilibrium field $\phi(\mathbf{r}, t)$ the quantity $\phi(\mathbf{r}, t)-\langle\phi(\mathbf{r}, t)\rangle$

\footnotetext{
*Electronic address: edgar.martin@staff.uni-marburg.de $\dagger$ Electronic address: ulrich.behn@itp.uni-leipzig.de URL: www.physik.uni-leipzig.de/ behn

‡Electronic address: guido.germano@staff.uni-marburg.de URL: www.uni-marburg.de/fb15/ag-germano
}

does not change sign. The nonequilibrium field can also be a scalar or tensorial order parameter field. Yurke et al. 7] have measured the probability that the local order parameter in a twisted nematic liquid crystal system has not switched its state up to a time $t$. Persistence phenomena have also been studied in the context of phase-ordering dynamics [8, 9], diffusion fields [10], and reaction-diffusion systems [11]. All these systems share the characteristic property that persistence, and hence also the distribution of first-passage times, follows a power law with some non-trivial exponent. However, in the literature that we have read, the reference point with respect to which persistence was measured has always been zero. In this work we shall consider the first-passage or first-exit problem with respect to a certain level $b$ for a stochastic process that may or may not be able to cross the origin, depending on the nature of the boundary at zero.

Our model can be described by the stochastic differential equation

$$
d X_{t}=\frac{n D}{X_{t}} d t+\sqrt{2 D} d W_{t},
$$

where $W_{t}$ is the Wiener process with zero mean

$$
\left\langle W_{t}\right\rangle=0,
$$

and the autocovariance function

$$
\left\langle W_{t} W_{t^{\prime}}\right\rangle=\min \left(t, t^{\prime}\right),
$$

the constant diffusion coefficient $D$ is positive, and the real parameter $n$ controls the relative strength of the drift and diffusion terms of the model.

Except for $n=0$ the origin $X_{t}=0$ is a singular point, the nature of which depends on the value of $n$. Intuitively one might think that the stochastic process cannot cross the origin for a non-zero $n$ : it should be bounded to the interval $(0, \infty)$ or $(-\infty, 0)$ depending on the initial value $x_{0}$ of the process. As we shall see later, this is only true for a certain range of $n$. 
For $n=0$ the process is nothing but the Wiener process. The probability density function (PDF) of the firstpassage time of a certain level $b$ at time $T$ starting at $x_{0}$ is well known,

$$
f(T)=\frac{\left|b-x_{0}\right| T^{-3 / 2}}{\sqrt{4 \pi D}} \exp \left[-\frac{\left(b-x_{0}\right)^{2}}{4 D T}\right] .
$$

This result can be derived, for example, in an elegant way by a simple scaling argument [12]. For long times one obtains from Eq. (4) a power law $f(T) \propto T^{-3 / 2}$.

For nonzero $n$ the situation is not so simple any more. For $n<1$ Bray [13] obtained a result for the PDF of the first time to hit the origin, which for long times is a power law too, $f(T) \propto T^{-(3-n) / 2}$; see also below. The persistence probability is then simply the probability that no hit has occurred in the time span $T$, that is $1-\int_{0}^{T} f\left(T^{\prime}\right) d T^{\prime}=\int_{T}^{\infty} f\left(T^{\prime}\right) d T^{\prime}$ and is again a power law, with exponent $-(1-n) / 2$.

In this paper we consider a more general problem. We ask for the first time to leave the interval $(0, b)$ either by crossing the upper boundary $b$ or by hitting the origin, if the nature of the singularity allows the latter event. Correspondingly we calculate the first-passage time distribution with respect to the upper bondary $b$ or the first-exit time distribution for the interval $(0, b)$.

The PDF is determined as the solution of a boundary value problem, which is a Sturm-Liouville eigenvalue problem. On the semi-infinite interval $(0, \infty)$ the problem has a continuous spectrum, whereas on the finite interval $(0, b)$ the spectrum is discrete.

The paper is organized as follows. In Sec. II we shortly explain the scientific relevance of our process and discuss its relation to other model processes. The main results of the paper are presented in Secs. III andIV In Sec. III we analyze the nature of the singular point at the origin, which depends on the value of $n$. First we adopt a heuristic approach by Bray [13], then we present a more sophisticated analysis following a scheme proposed by Feller [14]. In Sec. IV] we calculate the PDFs of the first-passage time or of the first-exit time; we derive the backward Kolmogorov equation, formulate the boundary value problem, which is of Sturm-Liouville type, and give the general solutions for different ranges of $n$. We conclude Sec. IV giving a short description of a numerical simulation method and comparing the analytical results with those from simulation.

\section{PHYSICAL MOTIVATION AND RELATED MODELS}

First we remark that Eq. (1), setting $n=d-1-U / D$, governs the dynamics of the radial component of the position of a random walker in a $\operatorname{logarithmic}$ potential $U \log x$ in dimension $d$ or of a free random walker in an effective dimension $d^{\prime}=d-U / D$, which may have noninteger values [13]. In this context it appears natural to assume $x>0$, and for free diffusion $U=0$ to restrict to $n>-1$.
Eq. (1) appears in various physical, chemical and biological problems. The context in which the relevance of Eq. (1) arises will now be explained starting from generic considerations and then proceeding further with specific physical problems. Godrèche and Luck [15] introduced a classification of stochastic processes into a group with "narrow" distributions, where all moments are finite, and "broad" distributions, where PDFs exhibit a power-law decay, and hence only a finite number of moments converge. The PDFs of the persistence, and therewith also of the first-passage time, will decay respectively either faster than any power law or algebraically, depending on the nature of the process imposed by its distribution.

The motion of atoms in a one-dimensional optical lattice formed by two counterpropagating laser beams with linear perpendicular polarization was studied by a similar equation in the high momentum region, where the momentum takes the role of the stochastic variable $x$ [16].

The Barkhausen noise was described phenomenologically by a model where the domain wall velocity as a function of the magnetization is also described by a similar Langevin equation if the demagnetizing factor is neglected [17, 18]. The magnetization takes the role of time.

Fogedby and Metzler, the former of which had already analyzed the generic Langevin equation before [19, 20], have applied the model to study the variable size of a DNA "bubble" 21], which emerges when at a certain temperature hydrogen bonds connecting base pairs from the opposite strands of the double helix are broken. The bubble size is measured by the number of broken bonds; in a continuum limit the discrete number of broken bonds can be replaced by a continuous variable $x$ and, according to the Poland-Scheraga model [22], the free energy of the system can be approximated for small bubble sizes as

$$
\mathcal{F} \approx c k_{\mathrm{B}} T \log x,
$$

where $c$ is a positive constant. Equilibrium is reached for a minimum of the free energy and the dynamics follows the Langevin equation

$$
\frac{d x}{d t}=-D \frac{d \mathcal{F}}{d x}+\xi(t)
$$

where $\xi(t)$ is a thermal noise assumed to be Gaussian with autocovariance $\left\langle\xi(t) \xi\left(t^{\prime}\right)\right\rangle=2 D k_{\mathrm{B}} T \delta\left(t-t^{\prime}\right)$.

A similar model was employed studying the translocation of a polymer through a pore, where the number of monomers on one side is chosen as the "translocation coordinate" [23].

An interesting application of the model was found by Bray [13], who showed that persistence and nonequilibrium critical dynamics are related in the context of the two-dimensional $X Y$-model with non-conserved order parameter, where the critical temperature is the temperature $T_{\mathrm{KT}}$ of the Kosterlitz-Thouless phase transition. The dynamics of a vortex-antivortex pair can be mapped to a one-dimensional Langevin equation corresponding to Eq. (1) by a series of transformations. 
It is impossible for us to discuss comprehensively all the publications dealing with similar models because too many of them exist. The list becomes even longer considering Eq. (11) as a special case of several different more general types of stochastic process. 25],

On the one hand it is a special Rayleigh process 24,

$$
d X_{t}=\left(\mu_{-1} X_{t}^{-1}+\mu_{1} X_{t}\right) d t+\sigma d W_{t},
$$

where $\mu_{-1}, \mu_{1}$ and $\sigma$ are constants. Setting $\mu_{-1}=n D$, $\mu_{1}=0$ and $\sigma=\sqrt{2 D}$ results in Eq. (1), while setting $\mu_{-1}=0$ reproduces the radial Ornstein-Uhlenbeck process in one dimension [26]. Using methods of classical Lie group symmetry analysis, it was shown that the Rayleigh process belongs to a maximal invariance group with six parameters whose member equations can be reduced to the standard diffusion equation by appropriate changes of variables, thus leading to the analytical solutions of these equations 27 29].

The Rayleigh process is widely employed in physics, economics, finance, and other fields, e.g. biometry [30]. In physics, Eq. (7) appears e.g. within non-abelian gauge theories in the framework of stochastic quantization 31]. In economics, Eq. (7) appears e.g. as a special case of a more general diffusion process whose stationary solution has been proposed to model the distribution of the profit rate of firms [32]. In finance, the applications usually employ the form of the generalized Bessel process, which is introduced below.

On the other hand our process can be mapped onto the Bessel process

$$
d Y_{t}=a d t+b \sqrt{Y_{t}} d W_{t}
$$

via the transformation $Y_{t}=X_{t}^{2}$ : multiplying Eq. (11) with $X_{t}$, interpreting the stochastic integral in the Itō sense and using Itō's lemma, one recovers Eq. (8) with $a=2 \mu_{-1}+\sigma^{2}=2(n+1) D$ and $b=2 \sigma=\sqrt{8 D}$, where we have assumed $Y_{t} \geq 0$.

In finance, extensions of the Black-Scholes-Merton (BSM) option pricing formula [33, 34] based on diffusion processes where the volatility is a function of the underlying, called constant elasticity of variance or Cox processes [35], reduce to a more general Bessel process with an additional term proportional to $Y_{t}$ in the drift, corresponding to the Raleigh process, by means of a non-linear transformation and a measure change. The generalized Bessel process

$$
d Y_{t}=\left(a_{0}+a_{1} Y_{t}\right) d t+b Y_{t}^{\beta} d W_{t}
$$

describes the underlying stock price in the BSM lognormal model with $\beta=1, a_{0}=0$ and $a_{1}>0$, the short interest rate in the Vasicek model [36] with $\beta=0, a_{0}>0$ and $a_{1}<0$, and the short interest rate in the CoxIngersoll-Ross short rate model [37] with $\beta=1 / 2, a_{0}>0$ and $a_{1}<0$ (when $a_{1}<0$ the process is called meanreverting). These models with three adjustable parameters are all solvable. A general solution was proposed for a larger family of models with up to seven parameters; it has a similar structure as the BSM formula, the most notable difference being that error functions are replaced by confluent hypergeometric functions [38].

Eq. (9) is used to describe the underlying with $\beta=1$ or $\beta=1 / 2$ and $a_{0}=0$ also when pricing path-dependent options, e.g. barrier and lookback [39 42] or Asiatic options [43]. In this context the cumulative probability distribution $F(T)$ of the first-passage times of an upper barrier, i.e. the probability that the barrier is reached within a time $T$, is the probability that an up-and-in, or knock-in, option is valid at its maturity $T$ (here the barrier is an entry point), while $1-F(T)$ is the probability that an up-and-out, or knock-out, option is valid at $T$ (here the barrier is an exit point). In both cases a valid barrier option behaves as a European option; thus $F(T)$ is the probability that at maturity an up-and-in option behaves as a European option, and $1-F(T)$ is the probability that at maturity an up-and-out option behaves as a European option. These considerations lead to one approach (among others) to price barrier options.

\section{THE NATURE OF THE SINGULAR POINT AT THE ORIGIN}

The quantity we are interested in is the first-passage time with respect to a certain level $b$, when the initial value $x$ satisfies $0<x<b$. Clearly, the upper limit of the process is the artificially set absorbing boundary at $x=b$. It requires some effort to understand the nature of the lower boundary $x=0$, which is a singular point of the stochastic differential equation. We shall first adopt the heuristic argumentation by Bray [13] and then apply a more sophisticated classification scheme proposed by Feller [14].

\section{A. Heuristic arguments}

The Fokker-Planck equation corresponding to the stochastic differential equation (11) is

$$
\frac{\partial p(x, t)}{\partial t}=-\frac{\partial}{\partial x}\left[\frac{n D}{x} p(x, t)\right]+D \frac{\partial^{2} p(x, t)}{\partial x^{2}} .
$$

This does not depend on whether Eq. (11) is interpreted in the Itō or Stratonovich sense because in our case the noise is additive, i.e. independent of $x$ [44]. We restrict to $x \geq 0$, the case $x \leq 0$ being symmetric to the previous one. The general solution of the Fokker-Planck equation (10) requires the knowledge of two linearly independent solutions. Using the separation ansatz [13]

$$
p(x, t)=x^{(1+n) / 2} R_{k}(x) e^{-D k^{2} t}
$$

one gets the Bessel differential equation

$$
\frac{d^{2} R_{k}}{d x^{2}}+\frac{1}{x} \frac{d R_{k}}{d x}+\left(k^{2}-\frac{\nu^{2}}{x^{2}}\right) R_{k}=0
$$


where $\nu=(1-n) / 2$, whose solutions are the Bessel functions of the first kind $J_{\nu}(k x)$ and of the second kind $Y_{\nu}(k x)$.

For non-integer $\nu$ also $J_{\nu}(k x)$ and $J_{-\nu}(k x)$ are linearly independent, and we can use $J_{-\nu}$ instead of $Y_{\nu}$. In this case the general solution of Eq. (10) can be written as

$$
\begin{array}{r}
p(x, t)=x^{1-\nu} \int_{0}^{\infty}\left[A(k) J_{\nu}(k x)+\right. \\
\left.B(k) J_{-\nu}(k x)\right] \\
\times e^{-D k^{2} t} d k,
\end{array}
$$

where the coefficients $A(k)$ and $B(k)$ are to be determined by initial and boundary conditions.

The Bessel function of the first kind is given by [45]

$$
J_{\nu}(k x)=\sum_{l=0}^{\infty} \frac{(-1)^{l}}{l ! \Gamma(l+\nu+1)}\left(\frac{k x}{2}\right)^{2 l+\nu} .
$$

Denoting the contributions to $p(x, t)$ coming from $J_{\nu}$ and $J_{-\nu}$ by $p_{\nu}(x, t)$ and $p_{-\nu}(x, t)$ respectively, we can use Eq. (14) to write Eq. (13) in the form

$$
\begin{aligned}
p(x, t) & =p_{\nu}(x, t)+p_{-\nu}(x, t) \\
& =\sum_{l=0}^{\infty}\left[c_{\nu}^{l}(t) x^{2 l+1}+c_{-\nu}^{l}(t) x^{2 l+n}\right],
\end{aligned}
$$

with

$$
\begin{aligned}
c_{\nu}^{l}(t) & =\int_{0}^{\infty} A(k) \frac{(-1)^{l}(k / 2)^{2 l+\nu}}{l ! \Gamma(l+\nu+1)} e^{-D k^{2} t} d k \\
c_{-\nu}^{l}(t) & =\int_{0}^{\infty} B(k) \frac{(-1)^{l}(k / 2)^{2 l-\nu}}{l ! \Gamma(l-\nu+1)} e^{-D k^{2} t} d k .
\end{aligned}
$$

The behaviour of the PDF for $x \rightarrow 0^{+}$is determined by the leading order terms and thus, approaching zero, we find

$$
p(x, t) \sim c_{\nu}^{0}(t) x+c_{-\nu}^{0}(t) x^{n} .
$$

For $1<n$ the leading order term is $c_{\nu}^{0}(t) x$ and the next to leading order term is $c_{-\nu}^{0}(t) x^{n}$. For $-1<n \leq 1$ it is vice versa. For $n \leq-1$ the contribution from $J_{-\nu}$ has a non-normalizable singularity at the origin; see also below. Introducing the probability current density

$$
j(x, t)=D\left(\frac{n}{x}-\frac{\partial}{\partial x}\right) p(x, t),
$$

the Fokker-Planck equation (10) can be written in the form

$$
\partial_{t} p+\partial_{x} j=0 .
$$

Terms proportional to $x^{n}$ do not contribute to $j(x, t)$, and so we arrive at

$$
j(x, t) \sim c_{\nu}^{0}(t) D(n-1),
$$

which holds in leading order for $x \rightarrow 0^{+}$.
For $1<n$ the coefficient $c_{\nu}^{0}(t)$ is positive because in this case $c_{\nu}^{0}(t)=\lim _{x \rightarrow 0^{+}} p(x, t) / x$, and correspondingly $j(x, t)>0$ near the origin.

For $-1<n \leq 1$ this is not true in general, see Eq. (17). The characterization can be made clearer imposing specific boundary conditions.

For example, absorbing boundary conditions at $x=$ 0 require $\lim _{x \rightarrow 0^{+}} p(x, t)=0$ and $\lim _{x \rightarrow 0^{+}} j(x, t)<0$. For $-1<n \leq 0$ the former condition can be fulfilled only setting $B(k)=0 . A(k)$ is determined by the initial condition. Observing the orthogonality relation [46]

$$
\delta(\alpha-\beta)=\alpha \int_{0}^{\infty} k J_{\nu}(\alpha k) J_{\nu}(\beta k) d k,
$$

which holds for $\nu>-1 / 2$, i.e. for $n<2$, we see that choosing $A(k)$ such that

$$
p(x, t)=x^{1-\nu} x_{0}^{\nu} \int_{0}^{\infty} k J_{\nu}\left(k x_{0}\right) J_{\nu}(k x) e^{-D k^{2} t} d k
$$

fulfills the initial condition $p(x, 0)=\delta\left(x-x_{0}\right)$.

The integral in Eq. (22) can be explicitly evaluated [47] with the result

$$
p(x, t)=x^{1-\nu} x_{0}^{\nu} \frac{1}{2 D t} \exp \left(-\frac{x^{2}+x_{0}^{2}}{4 D t}\right) I_{\nu}\left(\frac{x x_{0}}{2 D t}\right),
$$

given already by Bray [13]. Here $I_{\nu}$ is the modified Bessel function of the first kind defined by

$$
I_{\nu}(x)=\sum_{k=0}^{\infty} \frac{1}{k ! \Gamma(k+\nu+1)}\left(\frac{x}{2}\right)^{\nu+2 k} .
$$

Since in this case $B(x)=0$, which implies that also $c_{-\nu}=0$, we have $\lim _{x \rightarrow 0^{+}} p(x, t) / x=c_{\nu}(t)>0$, and from Eq. (20) it follows that $j\left(0^{+}, t\right)<0$ as required.

Note that the case of free diffusion $(n=0$, i.e., $\nu=$ $1 / 2$ ) with an absorbing boundary condition imposed at the origin is included in Eq. (23). A short calculation gives the well known result which can be obtained, e.g., by the mirror method.

As already shown by Karlin and Taylor [48], for $n \leq 1$ total absorption at the origin occurs in finite time. Correspondingly, in this case there exists a stationary solution of the Fokker-Planck equation which is a Dirac delta function $\delta(x)$; see also Alfarano et al. [32]. Formally this can be seen as follows. Observe that Eq. (10) admits stationary solutions $\tilde{p}_{\mathrm{s}}(x) \propto x^{n}$ whith $n \leq 1$ that are not normalizable at the origin. Using the concept of weak normalization introduced by Senf et al. 49], it can be shown that the weakly normalized version of $\tilde{p}_{\mathrm{s}}(x)$ is just a Dirac delta distribution, $p_{\mathrm{s}}^{\mathrm{w}}(x)=\delta(x)$, in the sense that $\int_{\mathrm{S}} p_{\mathrm{S}}^{\mathrm{w}}(x) \varphi(x) d x=\varphi\left(x_{0}\right)$, where $\varphi(x)$ is a test function and $x_{0}$ is included in the support $S$.

More insight into the qualitative behaviour of the system near and at the origin is provided by the classification scheme of Feller which is discussed in the next subsection. 


\section{B. Formal classification}

The modern classification of the boundaries of diffusion processes has been developed by Feller [14] and is based on semigroup operator arguments. We shall now briefly review the necessary theory for the boundary classification employing the notation of Karlin and Taylor [48] in order to be able to classify the origin for our process.

In the following let $X_{t}$ be a process defined on the interval $I=(l, r)$, where the two endpoints can be both finite or infinite. Also let the process start at the initial value $X_{0}=x$, and $a$ and $b$ be two finite real numbers such that the inequality $l<a<x<b<r$ holds. We shall consider regular diffusion processes in the interior of $I$, i.e. processes for which the first-passage time $T_{y}$ with respect to an arbitrary level $y$ in the interior of $I$ is finite with a positive probability

$$
P\left(T_{y}<\infty \mid X_{0}=x\right)>0 .
$$

The three central quantities are

$$
\begin{aligned}
u(x) & =P\left(T_{b}<T_{a} \mid X_{0}=x\right), \\
v(x) & =\left\langle T^{*} \mid X_{0}=x\right\rangle, \\
w(x) & =\left\langle\int_{0}^{T^{*}} g\left(X_{s}\right) d s \mid X_{0}=x\right\rangle,
\end{aligned}
$$

where $g$ is an arbitrary functional of the stochastic process, and we have defined $T^{*}=T_{a, b}=\min \left\{T_{a}, T_{b}\right\}$. It can be shown [48] that under certain conditions these quantities satisfy the boundary value problems

$$
\begin{aligned}
& L u(x)=0, \quad u(a)=0, \quad u(b)=1, \\
& L v(x)=-1, \quad v(a)=0, \quad v(b)=0, \\
& L w(x)=-g(x), \quad w(a)=0, \quad w(b)=0,
\end{aligned}
$$

with the differential operator $L$ acting on a function $f(x)$ as follows:

$$
L f(x)=\mu(x) f^{\prime}(x)+\frac{1}{2} \sigma^{2}(x) f^{\prime \prime}(x) .
$$

The proof for $u(x)$ invokes the law of total probability and uses a Taylor expansion to the second order around $x$ of the functional $u\left(X_{h}\right)$ at a small instant of time $h$. The proof for $w(x)$ uses a similar procedure, and finally the case $v(x)$ follows as a special case of $w(x)$ by setting $g(x) \equiv 1$.

The differential operator given by Eq. (32) can be written as

$$
\begin{aligned}
L f(x) & =\frac{1}{2} \sigma^{2}(x)\left(\frac{2 \mu(x)}{\sigma^{2}(x)} f^{\prime}(x)+f^{\prime \prime}(x)\right) \\
& =\frac{1}{2 m(x)} \frac{d}{d x}\left[\frac{1}{s(x)} \frac{d f(x)}{d x}\right]
\end{aligned}
$$

with

$$
s(x)=\exp \left[-\int^{x} \frac{2 \mu(\xi)}{\sigma^{2}(\xi)} d \xi\right]
$$

(the lower integration boundary is not indicated because it is arbitrary) and the speed density

$$
m(x)=\frac{1}{\sigma^{2}(x) s(x)} .
$$

Introducing the scale function

$$
S(x)=\int^{x} s(\eta) d \eta
$$

and the speed function

$$
M(x)=\int^{x} m(\eta) d \eta
$$

Eq. (33) can be rewritten in the form

$$
L f(x)=\frac{1}{2} \frac{d}{d M(x)}\left[\frac{d f(x)}{d S(x)}\right] .
$$

The definitions given by Eqs. (34) naturally induce measures of closed intervals $J=[c, d]$ : the scale measure

$$
S[J]=S[c, d]=S(d)-S(c)=\int_{c}^{d} s(x) d x,
$$

and the speed measure

$$
M[J]=M[c, d]=M(d)-M(c)=\int_{c}^{d} m(x) d x .
$$

These measures are fundamental for the classification of diffusion processes. The scale measure for an infinitesimal interval $J=[x, x+d x]$ is written symbolically as $S[d x]=S(x+d x)-S(x)=d S(x)=s(x) d x$, and the same applies for the speed measure.

Then Eqs. (29) and (31) can be easily integrated first with respect to the speed measure and thereafter with respect to the scale measure. Using the notation introduced above, the solutions can be expressed in compact form as

$$
u(x)=\frac{S[a, x]}{S[a, b]}
$$

and herewith

$$
\begin{aligned}
w(x)=2\{ & u(x) \int_{x}^{a} S[\eta, b] g(\eta) d M(\eta) \\
& \left.+[1-u(x)] \int_{x}^{a} S[a, \eta] g(\eta) d M(\eta)\right\} .
\end{aligned}
$$

The solution of Eq. (30) follows again from the special case of $g(x) \equiv 1$ in Eq. (42).

In the following only those definitions relevant for our classification will be mentioned, and not every proof can be given in detail. The book by Karlin and Taylor [48] is excellent for a deeper understanding. For the classification of the left boundary $l$ of a process, the procedure is to regard $u(x)$ and $v(x)$ in the limit $a \rightarrow l$. An analogous 
approach is employed for the right boundary; however we shall only be interested in the left boundary, which in our case is the zero level.

The first definition which is important for the understanding of whether a boundary can be reached is the attractiveness. A left boundary is called attractive if $S(l, x]:=\lim _{a \rightarrow l} S[a, x]<\infty$ for some $x \in(l, r)$. If the scale measure $S(l, x]$ is finite for some $x \in(l, r)$, this is also true for all $x$ in this interval. Hence it follows directly from Eq. (41) that $P\left(T_{l} \leq T_{b} \mid X_{0}=x\right)>0$ for all $l<x<b<r$, i.e. there is a positive probability that the left boundary is reached before the level $b$ in the interior of the interval, provided that the former is finite.

The next question is whether a boundary is attainable in finite time. This can be measured by $\lim _{a \rightarrow l} v(x)$, which is the expectation value of the first exit time from the interval $(l, b)$. Provided that the boundary is attractive, and using the solution $v(x)$ given by setting $g(x) \equiv 1$ in Eq. (42), it can be shown that it suffices to check whether a certain functional called $\Sigma(l)$ is finite in order to establish the attainability of the boundary. Hence a left boundary is said to be attainable if it is attractive and the functional

$$
\Sigma(l):=\int_{l}^{x} S(l, \xi] d M(\xi)=\int_{l}^{x} M[\eta, x] d S(\eta)
$$

is finite, otherwise it is said to be unattainable. Similarily one can define

$$
N(l):=\int_{l}^{x} M(l, \xi] d S(\xi)=\int_{l}^{x} S[\eta, x] d M(\eta) .
$$

The classification of the left boundary of a process is based on whether the functionals $S(l, x], M(l, x], \Sigma(l)$, and $N(l)$ are finite or not. These functionals are not independent of each other and some combinations are impossible; for example an attainable boundary is always attractive.

Using Feller's terminology, four types of boundaries can be distinguished. A process can both enter or leave from a regular boundary. The criteria for a left boundary to be regular are $S(l, x]<\infty$ and $M(l, x]<\infty$. In the case of an exit boundary it is impossible to reach any interior state $b$ if the starting point approaches $l$. A boundary is an exit boundary if $\Sigma(l)<\infty$ and $M(l, x]=\infty$. An entrance boundary cannot be reached from the interior of the state space, but it is possible to consider processes beginning there. It suffices to show that $S(l, x]=\infty$ while $N(l)<\infty$ to prove that $l$ is an entrance boundary. Finally, a natural or Feller boundary can neither be reached in finite mean time nor be the starting point of a process, and the corresponding criteria are $\Sigma(l)=\infty$ and $N(l)=\infty$.

We are now able to classify the zero level of our process. The first step is to check the attractivity. The parameters determining our process are $\mu(x)=n D / x$ and $\sigma^{2}(x)=2 D$. Since the scaling function only depends on the upper integration limit, we can choose the lower limit in a convenient way such that

$$
s(\eta)=\exp \left(-\int_{1}^{\eta} \frac{n}{z} d z\right)=\eta^{-n} .
$$

Then the scale measure of interest is

$$
\begin{aligned}
S & (0, x]=\lim _{a \rightarrow 0} \int_{a}^{x} \eta^{-n} d \eta \\
& = \begin{cases}\frac{1}{1-n}\left(x^{1-n}-\lim _{a \rightarrow 0} a^{1-n}\right) & \text { for } n \neq 1, \\
\log x-\lim _{a \rightarrow 0} \log a & \text { for } n=1,\end{cases}
\end{aligned}
$$

and thus the origin is attractive $(S(0, x]<\infty)$ for $n<1$, and non-attractive $(S(0, x]=\infty)$ for $n \geq 1$.

The speed density of the process is

$$
m(\eta)=\frac{\eta^{n}}{2 D}
$$

and we can evaluate the speed measure of an interval $(0, x]$ as

$$
\begin{aligned}
M(0, x] & =\frac{1}{2 D} \lim _{a \rightarrow 0} \int_{a}^{x} \eta^{n} d \eta \\
& = \begin{cases}\frac{1}{2 D(n+1)}\left(x^{n+1}-\lim _{a \rightarrow 0} a^{n+1}\right) & \text { for } n \neq-1, \\
\frac{1}{2 D}\left(\log x-\lim _{a \rightarrow 0} \log a\right) & \text { for } n=-1 .\end{cases}
\end{aligned}
$$

Hence we have $M(0, x]<\infty$ for $n>-1$ and $M(0, x]=\infty$ for $n \leq-1$. We now have established the nature of the zero level for $n<1$ : if $n \leq-1$ the origin is an exit boundary and in the case $-1<n<1$ it is a regular boundary. A regular boundary in the origin is the most complicated case. Karlin and Taylor [48] describe a regular boundary as follows:

For a regular boundary a variety of boundary behaviour can be prescribed in a consistent way, including the contingencies of complete absorption or reflecting, elastic or sticky barrier phenomena, and even the possibility of the particle (path), when attaining the boundary point, waiting there for an exponentially distributed duration followed by a jump into the interior of the state space according to a specified probability distribution function. In the latter event, the process only exhibits continuous sample paths over the interior of the state space.

The last step is to compute $N(0)$ for the classification of the case $n \geq 1$. Using Eq. (44) we get

$$
\begin{aligned}
N(0) & =\int_{0}^{x} S[\eta, x] d M(\eta) \\
& =\int_{0}^{x}\left(\int_{\eta}^{x} s(\xi) d \xi\right) m(\eta) d \eta \\
& =\frac{1}{2 D} \int_{0}^{x}\left(\int_{\eta}^{x} \xi^{-n} d \xi\right) \eta^{n} d \eta .
\end{aligned}
$$


It is easy to show that this double integral is always finite, and thus the origin is an entrance boundary for $n \geq 1$.

Summarizing, the nature of the boundary at zero has the following behaviour: exit if $n \in(-\infty,-1]$, regular if $n \in(-1,1)$, and entrance if $n \in[1, \infty)$.

\section{FIRST-PASSAGE AND FIRST-EXIT TIMES}

\section{A. Heuristic approach to first-passage times}

In a somewhat heuristic approach the first-passage (or first-exit) time PDF to leave an interval $(l, r)$ can be written as $f(T)=-\dot{G}(T)$ [50], where

$$
G(T)=\int_{a}^{b} p(x, T) d x
$$

is the probability that the particle is at time $T$ in $(a, b)$ when it has started at zero time at $x_{0}$ and we have calculated the PDF $p(x, t)$ imposing absorbing boundary conditions on those boundaries where the particle can leave the interval. Writing the Fokker-Planck, or forward Kolmogorov, equation in the form $\partial_{t} p+\partial_{x} j=0$ we have readily

$$
\dot{G}(T)=-\left.j(x, T)\right|_{a} ^{b}
$$

If the upper boundary is a natural boundary at $\infty$, $j(\infty, T)=0$, and we are interested in hits at the origin we have $f(T)=-j(0, T)$. For our problem the probability current density at the origin has been calculated in Sec. III A, From Eqs. (20) and (23) one obtains

$$
f(T)=\frac{1}{\Gamma(\nu)}\left(\frac{x_{0}^{2}}{4 D}\right)^{\nu} T^{-(\nu+1)} \exp \left(-\frac{x_{0}^{2}}{4 D T}\right) .
$$

For long times this is a power law $f(T) \propto T^{-(3-n) / 2}[13]$.

The result was obtained solving the Fokker-Planck equation (10) on the semi-infinite interval $(0, \infty)$ with an absorbing boundary condition at $x=0$ and the initial condition at $x=x_{0}$, and it is restricted to $n<1$, i.e. $\nu>0$. The spectrum of this boundary value problem is continuous.

However, if we are interested in the first time to leave a finite interval, we have to solve a boundary value problem with, for example, absorbing boundary conditions at both ends of the interval which typically has a discrete spectrum. We find it preferable to adopt a more formal approach, based on the backward Kolmogorov equation. The boundary value problem can then be transformed to a canonical Sturm-Liouville problem and systematically solved.

\section{B. The backward Kolmogorov equation}

In this section we use a special Fokker-Planck technique proposed by Kearney and Majumdar 51] to obtain a differential equation for the first-passage time PDF in Laplace space. Their method is very powerful, because the boundary conditions can be easily established in Laplace space and the functional $V\left[X_{t}\right]$ can be chosen such that different relevant quantities can be computed. Therefore we present the application of this method to our problem in some detail.

Considering a stochastic process starting at $X_{0}=x$ governed by the stochastic differential equation (1), we are interested in the PDF $f\left(T_{b}, x\right)$ of the first-passage time $T_{b}$ with respect to a certain level $b$, i.e. the time when the process has reached the level $b$ for the first time. First of all we define an arbitrary functional $V\left[X_{t}\right]$ by

$$
T=\int_{0}^{T_{b}} V\left[X_{t}\right] d t
$$

$T$ can have several meanings; in the special case $V\left[X_{t}\right] \equiv$ 1 it is simply the first-passage time $T_{b}$. The strategy is to find a differential equation in Laplace space for $f(T, x)$. The Laplace transform of $f(T, x)$ with respect to $T$ is given by

$$
\begin{aligned}
\tilde{f}(s, x) & =\mathcal{L}_{T}[f(T, x)](s) \\
& =\int_{0}^{\infty} f(T, x) e^{-s T} d T=\left\langle e^{-s T}\right\rangle_{T},
\end{aligned}
$$

where $s \in \mathbb{C}$. Splitting the interval $\left[0, T_{b}\right]$ into a small interval $[0, \Delta t]$ and an interval $\left(\Delta t, T_{b}\right]$, we can expand the integral over the small interval to first order in $\Delta t$ :

$$
\int_{0}^{\Delta t} V\left[X_{t}\right] d t=V[x] \Delta t+o(\Delta t) .
$$

Thus Eq. (53) becomes

$$
T=V[x] \Delta t+\int_{\Delta t}^{T_{b}} V\left[X_{t}\right] d t=: T_{1}+T_{2} .
$$

Inserting Eq. (56) into Eq. (54) gives

$$
\begin{aligned}
\tilde{f}(s, x) & =\left\langle e^{-s T}\right\rangle_{T}=\left\langle e^{-s T_{1}} e^{-s T_{2}}\right\rangle_{T} \\
& =\int_{0}^{\infty} f(T, x) e^{-s T_{1}} e^{-s T_{2}} d T .
\end{aligned}
$$

If we split the interval $\left[0, T_{b}\right]$ as described above, we must take into account that we also split our trajectory in two, where the starting point of the second part, $y:=X_{\Delta t}=$ $x+\Delta x$, is random itself. Therefore the PDF takes the form

$$
\begin{aligned}
f(T, x) & =\int_{0}^{b} f\left(T_{1}, x\right) f\left(T_{2}, y\right) d y \\
& =\int_{-x}^{b-x} f\left(T_{1}, x\right) f\left(T_{2}, x+\Delta x\right) d(\Delta x) .
\end{aligned}
$$

Inserting this into Eq. (57) and taking into account that $T_{1}$ is constant, and hence $d T=d T_{2}$, we obtain

$$
\tilde{f}(s, x)=e^{-s V[x] \Delta t}\langle\tilde{f}(s, x+\Delta x)\rangle_{\Delta x},
$$


where the average is done over all realizations of $\Delta x$. With Taylor expansions around $x$ of $e^{-s V[x] \Delta t}$ to the first order and of $\tilde{f}(s, x+\Delta x)$ to the second order, Eq. (59) becomes

$$
\begin{aligned}
& \tilde{f}(s, x)=(1-s V[x] \Delta t) \\
& \times\left[\tilde{f}(s, x)+\frac{\partial \tilde{f}(s, x)}{\partial x}\langle\Delta x\rangle+\frac{1}{2} \frac{\partial^{2} \tilde{f}(s, x)}{\partial x^{2}}\left\langle\Delta x^{2}\right\rangle\right] .
\end{aligned}
$$

In a first order approach

$$
\Delta x=\frac{n D}{x} \Delta t+\sqrt{2 D} \Delta W_{t},
$$

where $\Delta W_{t}=W_{t+\Delta t}-W_{t}$, and thus, using Eq. (2),

$$
\langle\Delta x\rangle=\frac{n D}{x} \Delta t .
$$

Then the mean value of $\left\langle\Delta x^{2}\right\rangle$ is, making again use of the zero mean property of the Wiener process, as well as of its autocorrelation function given in Eq. (3),

$$
\left\langle\Delta x^{2}\right\rangle=2 D \Delta t+o(\Delta t) .
$$

Finally, putting $V\left[X_{t}\right] \equiv 1$, we get the desired backward Kolmogorov equation for the first passage time PDF in Laplace space:

$$
\frac{\partial^{2} \tilde{f}(s, x)}{\partial x^{2}}+\frac{n}{x} \frac{\partial \tilde{f}(s, x)}{\partial x}-\frac{s}{D} \tilde{f}(s, x)=0 .
$$

\section{Formulation of the boundary value problem}

We now proceed to the formulation of the boundary value problems corresponding to the solutions of the firstpassage time PDFs, distinguishing between the three classes of boundaries the origin can belong to, as discussed in Sec. III. On the right side we impose an absorbing boundary at $b$ : the first-passage time vanishes for $x \rightarrow b^{-}$, i.e., $f\left(T, x \rightarrow b^{-}\right)=\delta(T)$. Inserting this into Eq. (54) gives

$$
\lim _{x \rightarrow b^{-}} \tilde{f}(s, x)=1 .
$$

The simplest case is if the zero level is an entrance boundary, i.e. $n \geq 1$. Starting from an inititial value $X_{0}=x>0$, the zero level can never be reached, which corresponds to a reflecting wall at the origin. Applying standard arguments for reflecting boundaries [50], the corresponding boundary condition is

$$
\lim _{x \rightarrow 0^{+}} \frac{\partial \tilde{f}(s, x)}{\partial x}=0 .
$$

For $n \leq-1$ the origin is an exit boundary. This means that it is impossible to reach any interior point of the state space if the initial point approaches the origin. This means that we have an absorbing boundary corresponding to

$$
\lim _{x \rightarrow 0^{+}} \tilde{f}(s, x)=1,
$$

and the first-passage time with respect to $x=b$ will diverge. Instead of the first-passage time the analysis of the previous section resulting in the backward Kolmogorov equation (64) together with the boundary conditions (65) and (67) gives the first-exit time from the interval $(0, b)$.

In the case of a regular boundary, which happens for $-1<n<1$, the behaviour is the most complicated. The process can both reach and leave the boundary zero, which means that also zero crossings are possible and the support of the process is the whole real axis. The firstexit time from $(0, b)$ is again given by the same boundary condition problem as in the case of the exit boundary.

For the sake of simplicity we rename $\tilde{f}(s, x)=: y(x)$. Restricting the process to the positive half axis, our boundary value problem for the three kinds of boundary in the origin reads

$$
\begin{gathered}
y^{\prime \prime}(x)+\frac{n}{x} y^{\prime}(x)-\frac{s}{D} y(x)=0, \\
\mathbf{A y}(0)+\mathbf{B y}(a)=\mathbf{c},
\end{gathered}
$$

where

$$
\mathbf{y}(x)=\left(\begin{array}{c}
y(x) \\
y^{\prime}(x)
\end{array}\right), \quad \mathbf{B}=\left(\begin{array}{ll}
0 & 0 \\
1 & 0
\end{array}\right)
$$

An absorbing boundary at zero corresponds to

$$
\mathbf{A}=\left(\begin{array}{ll}
1 & 0 \\
0 & 0
\end{array}\right), \quad \mathbf{c}=\left(\begin{array}{l}
1 \\
1
\end{array}\right),
$$

whereas a reflecting boundary at zero corresponds to

$$
\mathbf{A}=\left(\begin{array}{ll}
0 & 1 \\
0 & 0
\end{array}\right), \quad \mathbf{c}=\left(\begin{array}{l}
0 \\
1
\end{array}\right) .
$$

Multiplying Eq. (68) with the integrating factor $\exp \left(\int \frac{n}{x} d x\right)$ leads to

$$
-\left(x^{n} y^{\prime}\right)^{\prime}=-\frac{s}{D} x^{n} y .
$$

This is the canonical Sturm-Liouville form 52]

$$
-\left(p y^{\prime}\right)^{\prime}+q y=\lambda w y,
$$

with $p(x)=x^{n}$, the weighting function $w(x)=x^{n}$, $q(x) \equiv 0$, and the spectral parameter $\lambda=-s / D$.

We now observe that $u:=y-1$ transforms the homogeneous problem (68) with inhomogeneous boundary conditions (69) into an inhomogeneous problem with homogeneous boundary conditions

$$
\begin{aligned}
& -\left(p u^{\prime}\right)^{\prime}=\lambda w u+\lambda w, \\
& \mathbf{A u}(0)+\mathbf{B u}(a)=0,
\end{aligned}
$$


where $\mathbf{u}(x)=\left(u(x), u^{\prime}(x)\right)^{\top}$, and the two possible choices of $\mathbf{A}$ and $\mathbf{c}$ correspond to the Dirichlet problem and the Dirichlet-Neumann problem, respectively. This is easier to solve, since it determines a self-adjoint operator $\mathcal{L}$ defined by

$$
\mathcal{L} u=\frac{1}{w}\left[-\left(p u^{\prime}\right)^{\prime}\right]
$$

in the weighted Hilbert space $H=L^{2}(J, w)$, where we have defined the open interval $J=(0, b)$. This operator is not to be confused with the Laplace transformation operator $\mathcal{L}_{T}$ in Eq. (54), which can be recognized from the index indicating the transformed variable.

This can be seen as follows. Let $u, v \in H$; then the inner product is given by $\langle u, v\rangle=\int_{0}^{b} \bar{u} v w d x$; taking into account that $u$ and $v$ satisfy the homogeneous boundary conditions (76), we get after integrating twice by parts

$$
\begin{aligned}
\langle u, \mathcal{L} v\rangle & =-\int_{0}^{b} \bar{u}\left(p v^{\prime}\right)^{\prime} d x \\
& =\left[p\left(v \bar{u}^{\prime}-\bar{u} v^{\prime}\right)\right]_{0}^{b}-\int_{0}^{b}\left(p \bar{u}^{\prime}\right)^{\prime} v d x \\
& =\langle\mathcal{L} u, v\rangle .
\end{aligned}
$$

Using the definition from Eq. (77) the boundary value problem given by Eqs. (68 69) can be simplified to

$$
\begin{aligned}
(\mathcal{L}-\lambda \mathbf{1}) u & =\lambda, \\
\mathbf{A u}(0)+\mathbf{B u}(b) & =0 .
\end{aligned}
$$

\section{Formal solution of the boundary value problem}

We now exploit the property that the homogeneous boundary value problem with homogeneous boundary conditions

$$
\begin{aligned}
(\mathcal{L}-\alpha \mathbf{1}) u & =0 \\
\mathbf{A u}(0)+\mathbf{B u}(b) & =0
\end{aligned}
$$

has nontrivial solutions $u_{k}$ with eigenvalues $\alpha_{k}, k \in \mathbb{N}$,

$$
\mathcal{L} u_{k}=\alpha_{k} u_{k} .
$$

Because $\mathcal{L}$ is self-adjoint, the eigenvalues $\alpha_{k}$ are real and the eigenfunctions $u_{k}$ form an orthonormal basis of $H$. Furthermore $\alpha_{k}>0$ holds, since $\alpha_{k}=\left\langle u_{k}, \mathcal{L} u_{k}\right\rangle$. Hence the solution $u$ of the inhomogeneous problem given by Eqs. (79) and (80) can be expressed through an expansion in this basis,

$$
u=\sum_{k=1}^{\infty} c_{k} u_{k}
$$

with $c_{k}=\left\langle u_{k}, u\right\rangle$. Inserting $u=1$ gives the normalization, $\sum_{k=1}^{\infty}\left\langle u_{k}, 1\right\rangle u_{k}=1$. The coefficients $c_{k}$ can be derived from Eq. (79):

$$
\left\langle u_{k}, \mathcal{L} u\right\rangle-\left\langle u_{k}, \lambda u\right\rangle=\left\langle u_{k}, \lambda\right\rangle .
$$

Again, making use of the definition of a self-adjoint operator, we can pull $\mathcal{L}$ into the first component of the inner product. Employing Eq. (83) we get

$$
c_{k}=\frac{\left\langle u_{k}, \lambda\right\rangle}{\alpha_{k}-\lambda} .
$$

The solution of the inhomogeneous problem reads

$$
u=\sum_{k=1}^{\infty} \frac{\left\langle u_{k}, \lambda\right\rangle}{\alpha_{k}-\lambda} u_{k} .
$$

Because the eigenfunctions $u_{k}$ do not depend on $\lambda=$ $-s / D$ and the Laplace transformation is a linear operation we obtain the inverse Laplace transform of $y=1+u$ as

$$
\begin{aligned}
y(T, x) & =\mathcal{L}_{s}^{-1}[y(s, x)](T) \\
& =\mathcal{L}_{s}^{-1}[1]+\sum_{k=1}^{\infty}\left\langle u_{k}, 1\right\rangle u_{k} \mathcal{L}_{s}^{-1}\left[\frac{\lambda}{\alpha_{k}-\lambda}\right] \\
& =\delta(T)+\sum_{k=1}^{\infty}\left\langle u_{k}, 1\right\rangle u_{k}\left[\alpha_{k} D e^{-\alpha_{k} D T}-\delta(T)\right] .
\end{aligned}
$$

Since the $u_{k}$ are normalized the two delta functions cancel out. Returning to our original notation, we write the final result for the first-passage time (or first-exit time when appropriate) PDF as

$$
f(T, x)=\sum_{k=1}^{\infty}\left\langle u_{k}, 1\right\rangle u_{k} \alpha_{k} D e^{-\alpha_{k} D T} .
$$

Of course, this PDF is normalized to 1: knowing that $\alpha_{k}>0$ we have

$$
\begin{aligned}
\int_{0}^{\infty} f(T, x) d T & =\sum_{k=1}^{\infty}\left\langle u_{k}, 1\right\rangle u_{k} \alpha_{k} D \int_{0}^{\infty} e^{-\alpha_{k} D T} d T \\
& =\sum_{k=1}^{\infty}\left\langle u_{k}, 1\right\rangle u_{k}=1
\end{aligned}
$$

We are now able to solve the specific boundary value problems for the three different kinds of boundaries at zero.

\section{E. Comparison of theory and simulation}

\section{Simulation method}

To simulate the process $X_{t}$ that fulfills Eq. (11), we have used the Euler-Maruyama method [53 [56], which in this case with an additive noise is identical to the higherorder Milstein method [54 56]. A generic autonomous stochastic differential equation

$$
d X_{t}=\mu\left(X_{t}\right) d t+\sigma\left(X_{t}\right) d W_{t}
$$


can be integrated between two successive times $t_{n}$ and $t_{n+1}$, giving

$$
X_{n+1}=X_{n}+\int_{t_{n}}^{t_{n+1}} \mu\left(X_{t}\right) d t+\int_{t_{n}}^{t_{n+1}} \sigma\left(X_{t}\right) d W_{t},
$$

where $X_{n}$ is short for $X_{t_{n}}$. The approximation of the integrands to their value in $t_{n}$,

$$
\begin{aligned}
& \mu\left(X_{t}\right) \approx \mu\left(X_{n}\right), \\
& \sigma\left(X_{t}\right) \approx \sigma\left(X_{n}\right),
\end{aligned}
$$

yields the Euler method for the Itō case, called EulerMaruyama [53],

$$
X_{n+1}=X_{n}+\mu\left(X_{n}\right) \Delta t+\sigma\left(X_{n}\right) \Delta W_{n},
$$

where $\Delta t=t_{n+1}-t_{n}$ and $\Delta W_{n}=W_{n+1}-W_{n} \sim$ $N(0, \Delta t) \sim \sqrt{\Delta t} N(0,1)$, i.e. $\Delta W_{n}$ it is a normal random variable with $\mathrm{PDF}$

$$
p(w)=\frac{1}{\sqrt{2 \pi \Delta t}} \exp \left(-\frac{w^{2}}{2 \Delta t}\right) .
$$

The Euler-Maruyama method has strong order of convergence $1 / 2$. The Milstein method raises this to 1 adding to the right-hand side of Eq. (94) the correction $\frac{1}{2} \sigma\left(X_{n}\right) \sigma^{\prime}\left(X_{n}\right)\left[\left(\Delta W_{n}\right)^{2}-\Delta t\right]$, where $\sigma^{\prime}\left(X_{n}\right)=$ $d \sigma(x) /\left.d x\right|_{x=X_{n}}$. However, for an additive noise this derivative vanishes and so here the correction is zero. Schemes with order higher than 1 contain further terms some of which are nonzero also for additive noise, though many cancel out with respect to the general case, called multiplicative [44], where $\sigma$ depends on $X_{t}$.

The approximation of the noise term as

$$
\sigma\left(X_{t}\right) \approx \frac{\sigma\left(X_{n}\right)+\sigma\left(X_{n+1}\right)}{2}
$$

or as

$$
\sigma\left(X_{t}\right) \approx \sigma\left(\frac{X_{n}+X_{n+1}}{2}\right)
$$

yields the corresponding method for the Stratonovich case; if $\sigma$ is continuous, both Eqs. (96) and (97) lead to the same limit for $\Delta t \rightarrow 0$. This results in an implicit method, where to compute $X_{n+1}$ it is required to estimate it before; the predictor-corrector approach where $X_{n+1}$ in Eq. (96) or (97) is approximated by Eq. (94) for the Itō case is known by the name of Euler-Heun or Heun [54, 55]. As already observed at the beginning of Sec. IIIA with respect to the Fokker-Planck equation, both the Itō and the Stratonovich convention lead to the same result when the noise is additive as here. Interestingly the Milstein scheme represents both the order 1 strong Itō-Taylor approximation and the order 1 strong Stratonovich-Taylor approximation, i.e. even in the multiplicative case it coincides for both kinds of stochastic integral.
In other words, the choice of $X_{t}$ within the discretization interval $\left[X_{n}, X_{n+1}\right]$ affects the outcome of the integration only as far as the dependence of the noise term $\sigma$ on $X_{t}$ is concerned, because the covariation of $X_{t}$ and of the Wiener process $W_{t}$ driving the stochastic integral is not zero, $\left[X_{t}, W_{t}\right] \neq 0$ [57] (unfortunately closed intervals and covariations share the same notation). The choice of $X_{t} \in\left[X_{n}, X_{n+1}\right]$ has no influence on the integration of the drift term $\mu$ with respect to $t$, and the choice of $t \in\left[t_{n}, t_{n+1}\right]$ does not matter for either $\mu$ or $\sigma$ if they depend on $t$, as $[t, t]=0$ and $\left[t, W_{t}\right]=0$; in the three latter cases the same limit results for $\Delta t \rightarrow 0$.

Eq. (94) can be implemented straightforwardly in code. However, measuring the first-passage time with respect to a certain level needs a further refinement, since there is a finite hitting probability during each discretized time interval $\Delta t$, and thus the first-passage time is overestimated. An analytic expression for the probability that the process hits the level $b$ during a discretization interval $\Delta t$ was found by Mannella [58]. If we introduce the abreviations $\mu_{n}=\mu\left(X_{n}\right), \mu_{b}=\mu(b)$ and $\mu_{b}^{\prime}=d \mu(x) /\left.d x\right|_{x=b}$, the hitting probability reads

$$
\begin{aligned}
& P(\text { hit })=\exp \left\{-\frac{\mu_{b}^{\prime}}{2 D\left(e^{2 \mu_{b}^{\prime} \Delta t}-1\right)}\right. \\
& \times\left[X_{n+1}-b+\left(X_{n}-b\right) e^{\mu_{b}^{\prime} \Delta t}-\frac{\mu_{b}}{\mu_{b}^{\prime}}\right]^{2} \\
&\left.+\frac{1}{4 D \Delta t}\left[X_{n+1}-\left(X_{n}+\frac{\mu_{n}+\mu_{n+1}}{2} \Delta t\right)\right]^{2}\right\} .
\end{aligned}
$$

We can now summarize the simulation algorithm. We draw a Gaussian random number $\Delta W_{n}$ using e.g. the Box-Muller method [59] and propagate the process $X_{n}$ by a time step $\Delta t$. If the propagated value exceeds the level $b$ for the first time, i.e. $X_{n+1}>b$, the process is terminated. Otherwise we check for missed hits in the discretization interval by drawing a uniformly distributed random number $U \in[0,1)$ and accepting the hitting hypothesis if $P$ (hit) $>U$; this fulfills the second terminating condition. In both cases the first-passage time is set to $t_{n}$, i.e. the value before the propagation.

In the case of an entrance boundary a further refinement of the simulation algorithm is possible. Knowing that the zero level can never be reached from the interior of the state space of the process, it is clear that negative values in the simulations must result from discretization errors. If this is the case we can reduce the time step until the propagated value of the process is positive.

\section{Entrance boundary}

As we know from the classification of the origin, we have an entrance boundary for $n \geq 1$ (i.e. $\nu \leq 0$ ). The general solution of the homogeneous differential equa- 
tion (81) is

$$
u(x)=x^{\nu}\left[A J_{\nu}(\sqrt{\alpha} x)+B Y_{\nu}(\sqrt{\alpha} x)\right],
$$

where $J_{\nu}$ and $Y_{\nu}$ are the Bessel functions of the first and second kind, respectively.

Exploiting $J_{\nu}^{\prime}(x)=J_{\nu-1}(x)-(\nu / x) J_{\nu}(x)$ and an analogous formula for $Y_{\nu}$ [46], we obtain the derivative

$$
u^{\prime}(x)=\sqrt{\alpha} x^{\nu}\left[A J_{\nu-1}(\sqrt{\alpha} x)+B Y_{\nu-1}(\sqrt{\alpha} x)\right] .
$$

The relevant boundary conditions for $\tilde{f}(s, x)$ given by Eqs. (65) and (66) transform to $u(b)=0$ (absorption at $x=b$ ) and $\lim _{x \rightarrow 0} u^{\prime}(x)=0$ (reflection at $x=0$ ), respectively.

To evaluate the eigenfunctions in the case of negative and integer $\nu$ one can use the symmetry relation [46]

$$
J_{-\nu}(z)=(-1)^{\nu} J_{\nu}(z),
$$

which holds for integer $\nu$, to see that the first term in Eq. (100) goes to zero for $x \rightarrow 0$, because its leading order term behaves as $x$. Since the Bessel functions of second kind diverge as $x \rightarrow 0$, the reflecting boundary condition can be fulfilled only if $B=0$.

The absorbing boundary condition at $x=b$ determines the eigenvalues of the problem by the requirement that $J_{\nu}\left(\sqrt{\alpha_{k}} b\right)=0$. Denoting the $k$ th zero of $J_{\nu}(x)$ by $j_{k}$ we thus have $u_{k}(x)=A_{k} x^{\nu} J_{\nu}\left(j_{k} x / b\right)$. The constant $A_{k}$ is determined by the condition $\left\langle u_{k}, u_{l}\right\rangle=\delta_{k l}$. Remember that the brackets denote the scalar product in the weighted Hilbert space with weigth $w=x^{n}$. Observing the orthogonality relation [45]

$$
\int_{0}^{b} J_{\nu}\left(j_{k} \frac{x}{b}\right) J_{\nu}\left(j_{l} \frac{x}{b}\right) x d x=\frac{1}{2} b^{2} J_{\nu+1}^{2}\left(j_{k}\right) \delta_{k l}
$$

one obtains $A_{k}=\sqrt{2} b^{-1} / J_{\nu+1}\left(j_{k}\right)$, so that

$$
u_{k}(x)=\sqrt{2} b^{-1} x^{\nu} J_{\nu}\left(j_{k} \frac{x}{b}\right) / J_{\nu+1}\left(j_{k}\right) .
$$

We can further compute [47]

$$
\left\langle u_{k}, 1\right\rangle=\frac{\sqrt{2} b^{1-\nu}}{j_{k}}\left[\frac{\left(j_{k} / 2\right)^{\nu-1}}{\Gamma(\nu) J_{\nu+1}\left(j_{k}\right)}-\frac{J_{\nu-1}\left(j_{k}\right)}{J_{\nu+1}\left(j_{k}\right)}\right] .
$$

For integer $\nu$ a recurrence relation $J_{\nu+1}(x)+J_{\nu-1}(x)=$ $2 \nu J_{\nu}(x) / x$ holds, which, evaluated at the $k$ th zero of $J_{\nu}$, delivers $J_{\nu-1}\left(j_{k}\right)=-J_{\nu+1}\left(j_{k}\right)$. Hence Eq. (104) simplifies to

$$
\left\langle u_{k}, 1\right\rangle=\frac{\sqrt{2} b^{1-\nu}}{j_{k}}\left[\frac{\left(j_{k} / 2\right)^{\nu-1}}{\Gamma(\nu) J_{\nu+1}\left(j_{k}\right)}+1\right] .
$$

For non-integer $\nu$ the Bessel functions $J_{\nu}$ and $J_{-\nu}$ are two linear independent solutions of Eq. (81), and it is more convenient to write the general solution as

$$
u(x)=x^{\nu}\left[A J_{\nu}(\sqrt{\alpha} x)+B J_{-\nu}(\sqrt{\alpha} x)\right] .
$$

Exploiting $J_{-\nu}^{\prime}(x)=-J_{1-\nu}(x)-(\nu / x) J_{-\nu}(x)$ [46], the derivative can be written as

$$
u^{\prime}(x)=\sqrt{\alpha} x^{\nu}\left[A J_{\nu-1}(\sqrt{\alpha} x)-B J_{1-\nu}(\sqrt{\alpha} x)\right] .
$$

In this case $x^{\nu} J_{\nu-1}(\sqrt{\alpha} x)$ diverges as $x \rightarrow 0$, and the left boundary condition requires $A=0$. The right boundary condition determines the eigenvalues similarly as in the previous case; it is required that $\sqrt{\alpha} b$ are the zeros $j_{k}$ of the Bessel function $J_{-\nu}$. Again the second constant is evaluated using Eq. (102). The normalized eigenfunctions are

$$
u_{k}(x)=\sqrt{2} b^{-1} x^{\nu} J_{-\nu}\left(j_{k} \frac{x}{b}\right) / J_{1-\nu}\left(j_{k}\right)
$$

with

$$
\left\langle u_{k}, 1\right\rangle=\sqrt{2} b^{1-\nu} j_{k}^{-1} .
$$

For completeness we prove that the eigenfunctions given by Eqs. (103) and (108) fulfill the normalization condition (90). Setting $x / b=z$, for integer $\nu$ Eqs. (103) and (105) yield

$$
\begin{aligned}
& \sum_{k=1}^{\infty}\left\langle u_{k}, 1\right\rangle u_{k}=z^{\nu} \sum_{k=1}^{\infty} \frac{2 J_{\nu}\left(j_{k} z\right)}{j_{k} J_{\nu+1}\left(j_{k}\right)}\left[\frac{\left(2 / j_{k}\right)^{1-\nu}}{\Gamma(\nu) J_{\nu+1}\left(j_{k}\right)}+1\right] \\
= & z^{\nu} \sum_{k=1}^{\infty}\left[\frac{\left(2 / j_{k}\right)^{2-\nu} J_{\nu}\left(j_{k} z\right)}{\Gamma(\nu) J_{\nu+1}^{2}\left(j_{k}\right)}+\frac{2 J_{\nu}\left(j_{k} z\right)}{j_{k} J_{\nu+1}\left(j_{k}\right)}\right]=1, \quad(110)
\end{aligned}
$$

where we have used the Fourier-Bessel expansions

$$
\begin{aligned}
z^{\nu} & =\sum_{k=1}^{\infty} \frac{2 J_{\nu}\left(j_{k} z\right)}{j_{k} J_{\nu+1}\left(j_{k}\right)} \\
z^{-\nu}-z^{\nu} & =\sum_{k=1}^{\infty} \frac{\left(2 / j_{k}\right)^{2-\nu} J_{\nu}\left(j_{k} z\right)}{\Gamma(\nu) J_{\nu+1}^{2}\left(j_{k}\right)} .
\end{aligned}
$$

Eq. (111) is found in Watson [45], Eq. (112) is proved in the appendix. For non-integer $\nu$ Eqs. (108), (109) and (111) yield

$$
\sum_{k=1}^{\infty}\left\langle u_{k}, 1\right\rangle u_{k}=z^{\nu} \sum_{k=1}^{\infty} \frac{2 J_{-\nu}\left(j_{k} z\right)}{j_{k} J_{1-\nu}\left(j_{k}\right)}=1 .
$$

Fig. 11 shows the analytical results obtained with Wolfram Mathematica 7.0 by truncating the sum in Eq. (89) after the first 200 terms, and normalized histograms generated with 10 million simulation runs done as explained in Sec.IVE1 The agreement is perfect. The CPU time needed for an analytical curve is a few seconds, while that for a histogram with 10 million runs, which is the number used for all histograms in this paper, ranges from a few minutes to two days, depending on the time step, the starting position, and the upper boundary $b$. We used the ran1 uniform random number generator 60] and the GNU $\mathrm{C}++$ compiler $(\mathrm{g}++)$ version 4.1.2 with the -O3 optimization option on a $2.2 \mathrm{GHz}$ AMD Athlon 64 "Winchester" processor with Fedora Core 7 Linux. 

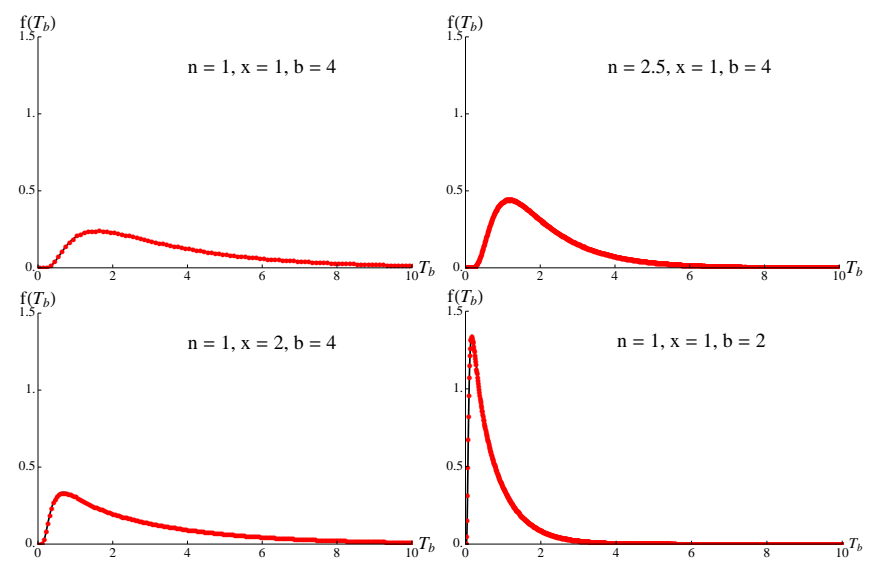

FIG. 1: First passage time PDF $f\left(T_{b}\right)$ when the origin is an entrance boundary. The parameter $n$, the starting position $x$, and the upper boundary $b$ are given in the insets; the diffusion coefficient $D$ is 1 . The analytical results (lines) are perfectly covered by the normalized histograms obtained from simulation (circles).

\section{Exit boundary}

For $n \leq-1$, i.e. $\nu \geq 1$, the zero level is an exit boundary and it is impossible to reach any interior point $b$, provided that the starting point of the process is sufficiently close to the boundary. This, repeating the arguments of Sec. IIIA corresponds to a collapse of the PDF to a delta function $\delta(x)$ in a finite time. Hence in general the first-passage time with respect to $x=b>0$ will diverge.

However, with the absorbing boundary at the origin, where naturally $u(0)=0$, and imposing an absorbing boundary condition at the upper boundary, $u(b)=0$, we have a boundary value problem with a solution that is the PDF of the first-exit time $T_{0, b}=\min \left\{T_{0}, T_{b}\right\}$ from the interval $(0, b)$.

The first terms in Eqs. (99) or (106) vanish in the limit $x \rightarrow 0$, whereas the second terms diverge. Therefore the constant $B$ must be zero in order to fulfill $u(b)=0$. As in the case of an entrance boundary, the eigenvalues are determined by the condition $J_{\nu}\left(\sqrt{\alpha_{k}} b\right)=0$ and the eigenfunctions are given by Eq. (103).

In Fig. 2 the theoretical curves are again compared with the results obtained numerically. It is interesting to notice that the PDF is bimodal for a range of parameters.

\section{Regular boundary}

For $-1<n<1$, i.e. $0<\nu<1$, the origin is a regular boundary, and in accordance with Karlin and Taylor [48] it is possible to impose different boundary conditions in a consistent way.

Imposing an absorbing boundary condition at the origin gives the PDF of the first-exit time. The eigenvalues are computed in the same way as for an exit boundary at the origin, and the eigenfunctions are again given

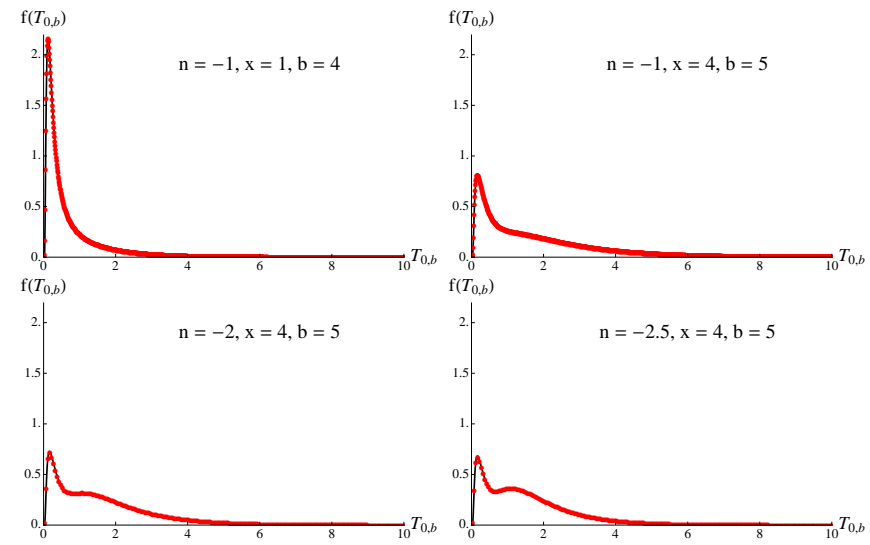

FIG. 2: First exit time PDF $f\left(T_{0, b}\right)$ when the origin is an exit boundary. The parameter $n$, the starting position $x$, and the upper boundary $b$ are given in the insets; the diffusion coefficient $D$ is 1 . The analytical results (lines) are perfectly covered by the normalized histograms obtained from simulation (circles).

by Eq. (103), which was also the result for the entrance boundary in the case of negative and integer $\nu$. Fig. 3 shows that the theoretical results agree perfectly with the simulations.
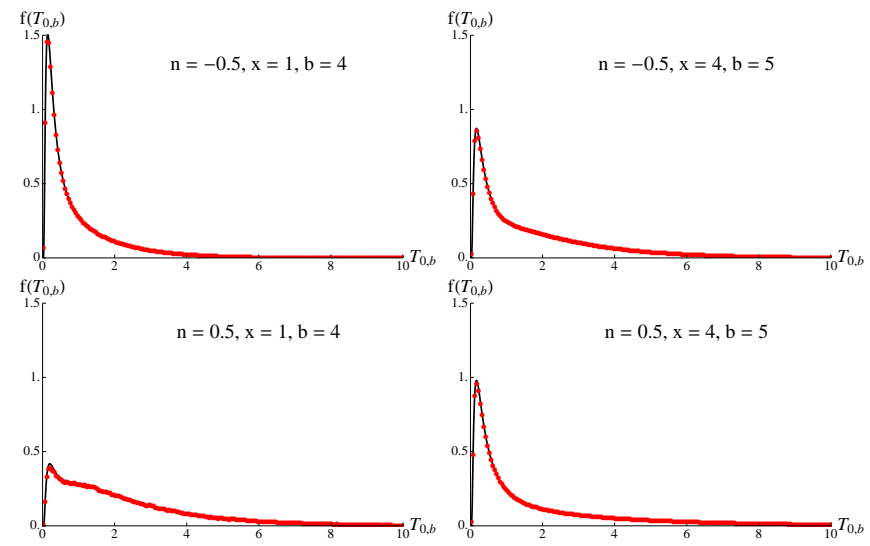

FIG. 3: First exit time PDF $f\left(T_{0, b}\right)$ when the origin is a regular boundary imposed to be absorbing. The parameter $n$, the starting position $x$, and the upper boundary $b$ are given in the insets; the diffusion coefficient $D$ is 1 . The analytical results (lines) are perfectly covered by the normalized histograms obtained from simulation (circles).

It is also possible to impose a reflecting boundary condition at the origin, and hence the eigenfunctions are computed in the same way as in the case of an entrance boundary, of course inserting the respective value of $n$. For a few values of $n$ in the range $0<n<1$ we have compared the theoretical curves resulting from the assumption that the origin is reflecting with histograms from simulations where we have allowed zero crossings; see the squares in Fig. 4. It appears that for small times there is a good agreement, whereas for larger times there 
are differences: the maxima of the histograms are higher than predicted by the theory, and the tails obtained by simulations are flatter than the theoretical tails. So we can clearly conclude that the origin is not naturally reflecting for this range of $n$, but, as one can see in Fig. 4. total reflection is approached when $n$ is approaching the limit where the origin is an entrance boundary, namely $n=1$.

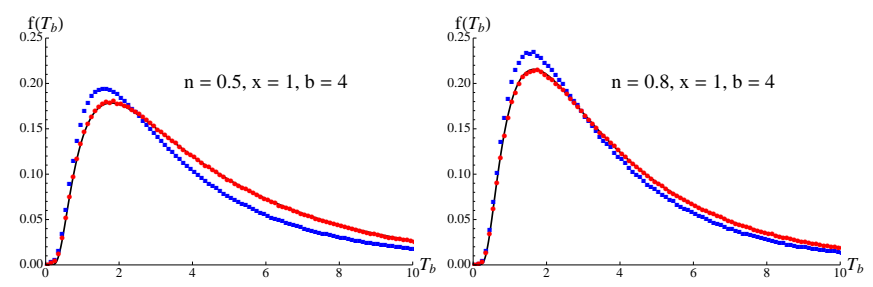

FIG. 4: First passage time PDF $f\left(T_{b}\right)$ when the origin is a regular boundary imposed to be reflecting. The parameter $n$, the starting position $x$, and the upper boundary $b$ are given in the insets; the diffusion coefficient $D$ is 1 . The analytical results (lines) are perfectly covered by the normalized histograms obtained from simulation (circles). Mismatching simulation results (squares) arise if zero crossings are allowed.

To explain this phenomenon we recall what we have mentioned earlier: one might think intuitively that zero crossings are not possible for non-zero values of $n$ since the drift term explodes near the origin, and the latter either reflects or absorbs the process for all times. However, applying Feller's formal classification scheme one can see that zero-crossings are actually allowed for a regular boundary at the origin, i.e. $-1<n<1$. This is further confirmed by Fig. 5 .

Knowing this and the fact that according to Karlin and Taylor [48] a process can spend a finite time in the vicinity of a regular boundary, we can explain the plots qualitatively. The paths that are able to escape the influence of the origin will quickly hit the boundary $b$ following the same rules as for the entrance boundary; they are basically driven by the drift term. The deviation in the tail of the PDFs is due to the positive amount of time spent in the vicinity of the origin, which is called the sticky boundary phenomenon [48], and to the multiple zero crossings. Fig. [6 shows a logarithmic plot of the first-passage time PDFs obtained by simulations, and one can see that the latter are heavy-tailed, i.e. they exhibit a power-law decay for long times. This is in contrast to the exponential decay obtained for the other types of boundary.

However, there is a good agreement between theory and simulation if we impose a reflecting origin in the simulation too, meaning that we consider the origin as a hard reflecting wall; see the circles in Fig. 4 .

For $-1<n<0$ the first-passage times diverge if we do not impose any artificial boundary condition, since the drift term is always negative if $X_{t}>0$ and positive if $X_{t}<0$, meaning that the process is always attracted, but not totally absorbed, by the origin. On the other hand,

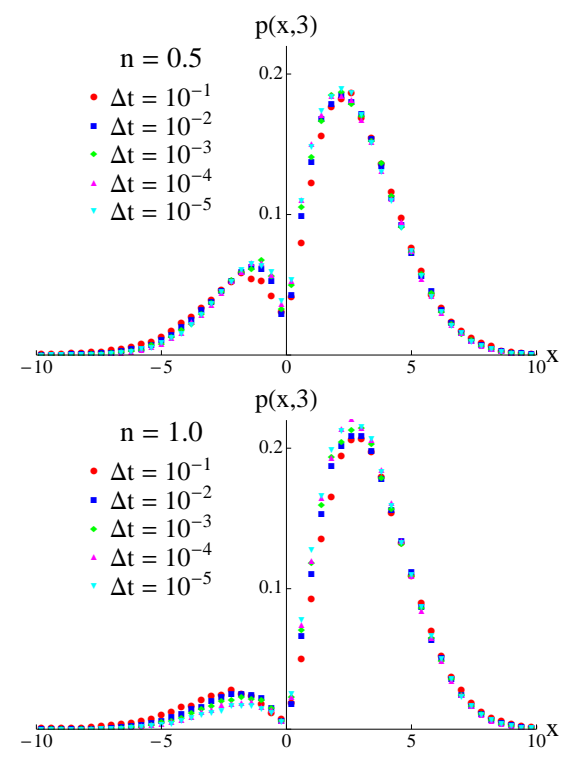

FIG. 5: PDFs of the stochastic process $X(t)$ at $t=3$ from simulation with different integration time step $\Delta t$ for $n=0.5$ (left) and $n=1.0$ (right); the starting position $x_{0}$ and the diffusion coefficient $D$ are 1 . For $n=0.5$ the peak in the negative domain increases with decreasing time step, whereas for $n=1$ it decreases. This suggests that in the latter case, where the origin is an entrance boundary, the zero crossings are an artifact due to the discretization of time, whereas in the former case, where the origin is regular, the zero crossings are genuine.

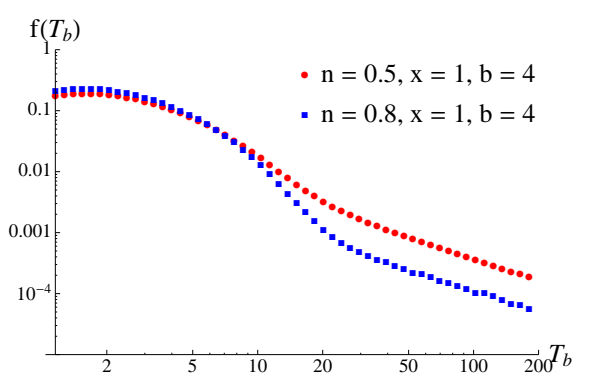

FIG. 6: Normalized histograms of first-passage times with respect to the level $b$ obtained by simulation when the origin is a regular boundary. The parameter $n$, the starting position $x$, and the upper boundary $b$ are given in the inset; the diffusion coefficient $D$ is 1 . The tails can be fitted by power laws with exponents -1.11 and -1.17 , respectively.

imposing total absorption at the origin corresponds to the computation of the PDF of the first-exit times as shown in Fig. 3.

It is interesting to note that the case $n=0$, i.e. the Wiener process, belongs to this class. The origin is not a singular point and the first-passage time PDF with respect to a level $b$ starting at $x_{0}$ is given by Eq. (4), which for long times is a power law with exponent $-3 / 2$. 


\section{CONCLUSIONS}

We have computed first-passage and first-exit time PDFs for a stochastic process with applications in many physical, chemical, biological, economical and financial problems. Depending on the nature of the boundary at the origin, we have found analytical solutions for the first-passage and first-exit time PDFs for all cases, except for the first-passage time PDF in the case of a regular boundary at the origin. In the latter case we have found an analytical solution for the first-exit time PDF and approximations for the first-passage time PDF for short times. For this specific stochastic process regularity of the boundary at zero can include behaviours ranging from total absorption to total reflection, with intermediate behaviours like elastic and sticky boundaries [48]. It is interesting that sticky boundaries may be applied e.g. to simulate the partial adsorption of polymer molecules to walls and for the modeling of solvent quality [61]. In possible future projects this could be investigated more thoroughly and regarded from the perspective of interactions between molecules and boundary surfaces, which is closely connected to another project of two of us 62, 63], where discotic liquid crystals confined in cylindric geometries 64] are studied via molecular dynamics simulations.

\section{Acknowledgements}

We thank R. Mannella, D. Marazzina, B. Schmitt, and A. Zettl for useful discussions and observations.

\section{Appendix}

We prove the Fourier-Bessel expansion given in Eq. (112). If a function $f(z)$ is represented in an orthogonal basis of Bessel functions of the first kind $J_{\nu}\left(j_{k} z\right)$, where $j_{k}$ is the $k$ th zero of $J_{\nu}(z)$, i.e. $J_{\nu}\left(j_{k}\right)=0$,

$$
f(z)=\sum_{k=1}^{\infty} c_{k} J_{\nu}\left(j_{k} z\right)
$$

and the orthogonality relation is given by Eq. (102), the $l$ th coefficient $c_{l}$ can be obtained from the scalar product of $f(z)$ with the $l$ th basis set element $J_{\nu}\left(j_{l} z\right)$,

$$
\begin{aligned}
\int_{0}^{1} J_{\nu}\left(j_{l} z\right) f(z) z d z & =\sum_{k=1}^{\infty} c_{k} \int_{0}^{1} J_{\nu}\left(j_{k} z\right) J_{\nu}\left(j_{l} z\right) z d z \\
& =\sum_{k=1}^{\infty} \frac{c_{k}}{2} J_{\nu+1}^{2}\left(j_{k}\right) \delta_{l k} \\
& =\frac{c_{l}}{2} J_{\nu+1}^{2}\left(j_{l}\right)
\end{aligned}
$$

resulting in

$$
c_{k}=\frac{2}{J_{\nu+1}^{2}\left(j_{k}\right)} \int_{0}^{1} J_{\nu}\left(j_{k} z\right) f(z) z d z=\frac{2 I_{k}}{J_{\nu+1}^{2}\left(j_{k}\right)}
$$

For $f(z)=z^{-\nu}$

$$
I_{k}=\int_{0}^{1} J_{\nu}\left(j_{k} z\right) z^{1-\nu} d z
$$

In order to exploit the equation 65]

$$
\int J_{\nu}(z) z^{1-\nu} d z=-J_{\nu-1}(z) z^{1-\nu}
$$

we substitute $j_{k} z=\alpha$ and get

$$
\begin{aligned}
I_{k} & =j_{k}^{\nu-2} \int_{0}^{j_{k}} J_{\nu}(\alpha) \alpha^{1-\nu} d \alpha \\
& =j_{k}^{\nu-2}\left[-J_{\nu-1}(\alpha) \alpha^{1-\nu}\right]_{0}^{j_{k}} \\
& =j_{k}^{\nu-2}\left[\lim _{\alpha \rightarrow 0} J_{\nu-1}(\alpha) \alpha^{1-\nu}-J_{\nu-1}\left(j_{k}\right) j_{k}^{1-\nu}\right]
\end{aligned}
$$

The limit is

$$
\begin{aligned}
\lim _{\alpha \rightarrow 0} \alpha^{1-\nu} J_{\nu-1}(\alpha) & =\lim _{\alpha \rightarrow 0} \alpha^{1-\nu} \sum_{l=0}^{\infty} \frac{(-1)^{l}}{l ! \Gamma(l+\nu)}\left(\frac{\alpha}{2}\right)^{2 l+\nu-1} \\
& =\sum_{l=0}^{\infty} \frac{(-1)^{l} 2^{1-\nu-2 l}}{l ! \Gamma(l+\nu)} \lim _{\alpha \rightarrow 0} \alpha^{2 l} \\
& =\frac{2^{1-\nu}}{\Gamma(\nu)},
\end{aligned}
$$

yielding

$$
I_{k}=j_{k}^{\nu-2}\left[\frac{2^{1-\nu}}{\Gamma(\nu)}-\frac{J_{\nu-1}\left(j_{k}\right)}{j_{k}^{\nu-1}}\right]
$$

Thus

$$
z^{-\nu}=\sum_{k=1}^{\infty}\left[\frac{\left(2 / j_{k}\right)^{2-\nu}}{\Gamma(\nu)}-\frac{2 J_{\nu-1}\left(j_{k}\right)}{j_{k}}\right] \frac{J_{\nu}\left(j_{k} z\right)}{J_{\nu+1}^{2}\left(j_{k}\right)}
$$

Subtracting the Fourier-Bessel expansion of $z^{\nu}$, Eq. (111), the second term in square braces cancels out because of the recurrence identity $J_{\nu-1}\left(j_{k}\right)+J_{\nu+1}\left(j_{k}\right)=2 \nu J_{\nu}\left(j_{k}\right) / j_{k}=0$ that we have already used to simplify Eq. (104) to Eq. (105). 
[1] E. W. Montroll and K. E. Shuler, Adv. Chem. Phys. 1, 361 (1958).

[2] B. J. Widom, J. Chem. Phys. 30, 238 (1959).

[3] F. H. Ree, T. S. Ree, T. Ree, and H. Eyring, Adv. Chem. Phys. 4, 1 (1962).

[4] T. Munakata and Y. Kaneko, Phys. Rev. E 47, 4076 (1993).

[5] S. Redner, A Guide to First-Passage Processes (Cambridge University Press, New York, 2001).

[6] S. N. Majumdar, Curr. Sci. 77, 370 (1999).

[7] B. Yurke, A. N. Pargellis, S. N. Majumdar, and C. Sire, Phys. Rev. E 56, R40 (1997).

[8] A. J. Bray, B. Derrida, and C. Godrèche, Europhys. Lett. 27, 175 (1994).

[9] S. N. Majumdar and C. Sire, Phys. Rev. Lett. 77, 1420 (1996).

[10] S. N. Majumdar, C. Sire, A. J. Bray, and S. J. Cornell, Phys. Rev. Lett. 77, 2867 (1996).

[11] E. Ben-Naim, Phys. Rev. E 53, 1566 (1996).

[12] S. C. Lim and S. V. Muniandy, Phys. Rev. E 66, 021114 (2002).

[13] A. J. Bray, Phys. Rev. E 62, 103 (2000).

[14] W. Feller, An Introduction to Probability Theory and Its Applications, vol. 2 (Wiley, New York, 1971), 3rd ed.

[15] C. Godrèche and J. M. Luck, J. Stat. Phys. 104, 489 (2001).

[16] E. Lutz, Phys. Rev. Lett. 93, 190602 (2004).

[17] S. Zapperi, P. Cizeau, G. Durin, and H. E. Stanley, Phys. Rev. B 58, 6353 (1998).

[18] F. Colaiori, S. Zapperi, and G. Durin, J. Magn. Magn. Mater. 272-276, e533 (2004).

[19] H. C. Fogedby and V. Poutkaradze, Phys. Rev. E 66, 021103 (2002).

[20] H. C. Fogedby, Phys. Rev. E 68, 051105 (2003).

[21] H. C. Fogedby and R. Metzler, Phys. Rev. E 76, 061915 (2007).

[22] D. Poland and H. A. Scheraga, Theory of Helix-Coil Transitions in Biopolymers (Academic Press, New York, 1970).

[23] J. Chuang, Y. Kantor, and M. Kardar, Phys. Rev. E 65, 011802 (2001).

[24] L. Rayleigh, Phil. Mag. Lett. 32, 473 (1902).

[25] V. Giorno, A. G. Nobile, L. M. Ricciardi, and L. Sacerdote, J. Appl. Probab. 23, 398 (1986).

[26] G. E. Uhlenbeck and L. S. Ornstein, Phys. Rev. 36, 823 (1930).

[27] V. Stohny, Nonlin. Math. Phys. 4, 132 (1997).

[28] S. Spichak and V. Stognii, J. Phys. A: Math. Gen. 32, 8341 (1999).

[29] K. Pesz, J. Phys. A: Math. Gen. 35, 1827 (2002).

[30] R. Gutiérrez, R. Gutiérrez-Sánchez, and A. Nafidi, Appl. Math. Comput. 175, 628 (2005).

[31] M. Horibe, A. Hosoya, and J. Sakamoto, Progr. Theor. Phys. 70, 1636 (1983).

[32] S. Alfarano, M. Milaković, A. Irle, and J. Kauschke, A statistical equilibrium model for competitive firms (2008), Christian-Albrechts-Universität Kiel, Economics Working Paper No. 2008-10, http://www.wiso.unikiel.de/Ordnung+Wettbewerbspolitik/ewp/ewp.php.

[33] F. Black and M. Scholes, J. Polit. Econ. 81, 637 (1973).
[34] R. C. Merton, Bell J. Econ. Manage. Sci. 4, 141 (1973).

[35] J. Cox, J. Portfolio Manage. 22, 15 (1996), reprint of Notes on option pricing I: constant elasticity of variance diffusions, Stanford University (1955).

[36] O. Vasicek, J. Financ. Econ. 5, 177 (1977).

[37] J. C. Cox, J. E. Ingersoll, and S. A. Ross, Econometrica 53, 385 (1985).

[38] C. Albanese, G. Campolieti, P. Carr, and A. Lipton, Risk, December 2001, page 99.

[39] D. Davydov and V. Linetsky, Manage. Sci. 47, 949 (2001).

[40] D. Davydov and V. Linetsky, Oper. Res. 51, 185 (2003).

[41] V. Linetsky, Finance Stochast. 8, 373 (2004).

[42] G. Fusai and M. C. Recchioni, J. Econ. Dyn. Control 31, 826 (2007).

[43] G. Fusai, M. Marena, and A. Roncoroni, J. Bank. Finance 32, 2033 (2008).

[44] R. F. Fox, J. Math. Phys. 13, 1196 (1972).

[45] G. N. Watson, A Treatise on the Theory of Bessel Functions (University Press, Cambridge, 1962), 2nd ed.

[46] G. Arfken, Mathematical Methods for Physicists (Academic Press, Orlando, 1985), 3rd ed.

[47] I. S. Gradshteyn and I. M. Rhyzhik, Table of Integrals, Series, and Products (Academic Press, New York, 1965).

[48] S. Karlin and H. M. Taylor, A Second Course in Stochastic Processes (Academic Press, New York, 1981), 2nd ed.

[49] F. Senf, P. M. Altrock, and U. Behn, New J. Phys. 11, 063010 (2009).

[50] C. W. Gardiner, Handbook of Stochastic Methods (Springer, Berlin, 2002), 2nd ed.

[51] M. J. Kearney and S. N. Majumdar, J. Phys. A: Math. Gen. 38, 4097 (2005).

[52] A. Zettl, Sturm-Liouville Theory (AMS, Providence, 2005).

[53] G. Maruyama, Rend. Circolo Mat. Palermo 4, 48 (1955).

[54] G. N. Milstein, Numerical Integration of Stochastic Differential Equations (Kluwer, Dordrecht, 1995).

[55] P. E. Kloeden and E. Platen, Numerical Solution of Stochastic Differential Equations (Springer, Berlin, 1999).

[56] D. J. Higham, SIAM Rev. 43, 525 (2001).

[57] G. Germano, M. Politi, E. Scalas, and R. L. Schilling, Phys. Rev. E 79, 066102 (2009).

[58] R. Mannella, Phys. Lett. A 254, 257 (1999).

[59] G. E. P. Box and M. E. Muller, Ann. Math. Stat. 29, 610 (1958).

[60] W. H. Press, S. A. Teukolsky, W. T. Vetterling, and B. P. Flannery, Numerical Recipes in $\mathrm{C}++$ (Cambridge University Press, Cambridge, 2003), 2nd ed.

[61] E. A. J. F. Peters and T. M. A. O. M. Barenbrug, Phys. Rev. E 66, 056702 (2002).

[62] C. Stillings, E. Martin, M. Steinhart, R. Pettau, J. Paraknowitsch, M. Geuss, J. Schmidt, G. Germano, H. W. Schmidt, U. Gösele, et al., Mol. Cryst. Liq. Cryst. 495, 285 (2008).

[63] E. Martin and G. Germano (2010), preprint.

[64] D. Caprion, Eur. Phys. J. E 28, 305 (2009).

[65] The Wolfram Functions Site, http://functions.wolfram.com. 